PROCEEDINGS OF THE

AMERICAN MATHEMATICAL SOCIETY

Volume 45, Number 1, July 1974

\title{
A SINGULAR PRIMITIVE RING
}

\author{
JOHN LAWRENCE ${ }^{1}$
}

ABSTRACT. An example of a primitive ring with nonzero singular ideal is constructed. An example, due to B. Osofsky, of a semiprimitive ring with nonzero singular ideal is shown to be nonprimitive.

All rings are associative with a unit element. All modules are unitary. $R$ is a (right) primitive ring if it has a faithful irreducible right module. $A$ right ideal of $R$ is essential if it has nontrivial intersection with every nonzero right ideal of $R$. The singular ideal $Z(R)$ is the set of elements of $R$ which annihilate essential right ideals on the left. Equivalently

$$
Z(R)=\{x \in R: \forall y(\neq 0) \in R, \exists z \in R \text { such that } y z \neq 0, x y z=0\} .
$$

The existence of a primitive ring with nonzero singular ideal has been an open problem for several years. In [3] Osofsky constructed an example of a semiprimitive ring with nonzero singular ideal. Both Osofsky and Faith $[1$, p. 128$]$ conjectured the existence of primitive $r$ ings with singular ideal. In this paper we construct such a ring, and also show that Osofsky's ring is not primitive.

In proving the primitivity of the ring, we use several ideas from [2]. In particular we use

Theorem 1. A ring is (right) primitive if and only if it has a proper right ideal M comaximal with every nonzero two-sided ideal of $R$; i.e. if $J(\neq 0)$ is a two-sided ideal, then $J+M=R$.

The ring which we will construct is very similar to Osofsky's example in [3].

Let $F=Z_{2}\left[X, Y_{j}\right], j=1,2, \cdots$, be the algebra over $Z_{2}$ in noncommuting variables. An arbitrary monomial in $F$ can be written as

Presented to the Society, August 22, 1973 under the title $A$ primitive ring with nonzero singular ideal; received by the editors July 31, 1973.

AMS (MOS) subject classifications (1970). Primary 16A20; Secondary 16A08.

Key words and phrases. Primitive ring, singular ring, singular ideal, semiprimitive ring.

${ }^{1}$ The author would like to thank Dr. I. Connell for his help and encouragement. 


$$
m=X^{i} Y_{j_{1}} X^{i_{2}} \quad \ldots \quad Y_{j_{n-1}} X^{i_{n}}, \quad i \geq 0, j \geq 1,
$$

repetitions allowed. Define

$$
\begin{aligned}
c(m) & =\sum i_{k}=\text { degree of } X \text { in } m, \\
d(m) & =\sum i_{k}+n-1=\text { degree of } m, \\
e(m) & =\max \left\{j_{k} \text { times the number of times } Y_{j_{k}} \text { appears }\right\}, \\
& =0, \quad \text { otherwise. }
\end{aligned}
$$

Let $I$ be the ideal of $F$ generated by monomials $m$ such that $c(m)>$ $e(m) \geq 1$. Let $R=F / I$. We claim that this is the desired example. $I$ is 'homogeneous' in the sense that a sum of distinct monomials of $F$ is in $I$ if and only if each monomial is in $l$. This allows us to speak of monomials in $R$. Given $(0 \neq) f=m_{1}+\cdots+m_{n}$, a sum of distinct nonzero monomials in $R$, define

$$
c(f)=\max \left\{c\left(m_{i}\right)\right\}, \quad d(f)=\max \left\{d\left(m_{i}\right)\right\}, \quad e(f)=\max \left\{e\left(m_{i}\right)\right\},
$$

Theorem 2. $Z(R) \neq(0)$.

Proof. This is similar to the proof of Lemma 4 in [3]. Suppose $(0 \neq)$ $f \in R$, a sum of monomials as above. Let $s=\min \left\{c\left(m_{i}\right)\right\}$. Choose $t>$ $\max \{d(f), e(f)\}$. Then $f Y_{t} X^{t-s} \neq 0$ and $X f Y_{t} X^{t-s}=0$; hence $X \in Z(R)$.

Since $R$ is countable, we can order the nonzero elements $f_{2}, f_{3}, \cdots$. We start the numbering at 2 in order to simplify certain subsequent statements. Given $f_{n}$ in this sequence, choose $j_{n}>1$ large enough so that $Y_{j_{n}} f_{n} Y_{j_{n}} \neq 0$, and let

$$
q_{n}=Y_{n} Y_{1}^{i} Y_{j_{n}} f_{n} Y_{j_{n}} Y_{1}^{i_{n}} \neq 0
$$

where $i_{n}>2 \max \left\{d\left(f_{n}\right), e\left(f_{n}\right)\right\}$. Choose $i_{n+1}>i_{n}, j_{n+1}>j_{n}$. Suppose $q_{n}=$ $a(n, 1)+a(n, 2)+\cdots+a\left(n, k_{n}\right)$, a sum of distinct nonzero monomials in $R$. Let $A$ be the subring of $R$ generated by all the $a(n, m)$.

Lemma. The following hold in $A$.

1. If $m_{1}, m_{2}$ are any monomials of $R$ and $a(n, i), a(m, j)$ are generators of $A$, then $0 \neq a(n, i) m_{1}=a(m, j) m_{2} \Rightarrow i=j, m=n$.

2. If $a_{1}, \cdots, a_{n}$ are generators of $A$, then $\Pi a_{i} \neq 0$.

3. $A$ is a free $Z_{2}$-algebra on the given generators.

4. If $a \in A$ and $b \in R$ and $b$ is a sum of monomials, none of which is in $A$, then $a b \in A \Rightarrow a b=0$. 
Proof. 1. Since the products are nonzero, they are equal if and only if they are identical. Since $a(n, i)$ begins with $Y_{n}$ and $a(m, j)$ begins with $Y_{m}$, we conclude that $m=n$. Now

$$
a(n, i) m_{1}=Y_{n} Y_{1}^{i_{n}} Y_{j_{n}} b Y_{j_{n}} Y_{1}^{i_{n}} m_{1}, \quad n \neq 1, j_{n} \neq 1 .
$$

As $i_{n}>d(b)$, we can 'decide' which generator occurs at the beginning of the product.

2. $\Pi a_{k}=\Pi Y_{k} Y_{1}^{2} Y_{j_{k}} b_{k} Y_{j} Y_{1}^{i} k$, where $i_{k}>2 \max \left\{d\left(b_{k}\right), e\left(b_{k}\right)\right\}$. If this product is zero then some segment of it must be a generator of $I$, say $m=$ $Y_{j_{s}} b_{s} Y_{j_{s}} Y_{1}^{i_{s}} \cdots Y_{t} Y_{1}^{i_{t}} Y_{j_{t}} b_{t} Y_{j_{t}}$. If $s \neq t$, then $c(m) \leq \Sigma_{k=s}^{t} c\left(b_{k}\right)$ and $e(m) \geq$ $\Sigma_{k=s}^{t} i_{s}$, and so $e(m)>c(m)$, a contradiction. If the generator contains only one $b_{k}$ (or part of one), the result is obvious.

3. This follows from 1 and 2. If $a_{i_{1}} \cdots a_{i_{k}}=a_{j_{1}} \cdots a_{j_{n}}$, then $2 \mathrm{im}$ plies that this product is nonzero, and using 1 inductively, we obtain $a_{i_{1}}=a_{j_{1}}$, etc.

4. Since both $I$ and $A$ are generated by monomials, we will have finished if we can prove the result assuming both $a$ and $b$ are monomials. Suppose $a=a_{i_{1}} \cdots a_{i_{k}}$ and $0 \neq a b=a_{j_{1}} \cdots a_{j_{n}}$. Using 1 , we conclude that $b \in A$, a contradiction. If $a=1$, the result is obvious.

Theorem 3. $R$ is primitive.

Proof. Let $M$ be the right ideal of $R$ generated by the set $\left\{q_{n}+1\right\}$, $n=2,3, \cdots$. If $(0) \neq J$ is a two-sided ideal of $R$, then $f_{n} \in J$, for some $n$, hence $q_{n} \in J$. Then $1 \in M+J$, hence $M$ is comaximal. If $M$ is not proper, then for some $\left\{r_{i}\right\} \subset R$ and some $n$, we have

$$
\left(q_{2}+1\right) r_{2}+\left(q_{3}+1\right) r_{3}+\cdots+\left(q_{n}+1\right) r_{n}=1 \text {. }
$$

Let $r_{i}=s_{i}+t_{i}$, where each monomial of $s_{i}$ is not in $A$ and each monomial of $t_{i}$ is in $A$. Then $\Sigma\left(q_{i}+1\right)\left(s_{i}+t_{i}\right)=1$ and so $\Sigma\left(q_{i}+1\right) s_{i}=0$, by the previous lemma, and $\Sigma\left(q_{i}+1\right) t_{i}=1$.

The latter equation gives a nontrivial relation in the free algebra $A$, and hence, is impossible. Since we arrive at a contradiction by assuming that $M$ is not proper, the theorem follows (use Theorem 1).

We conclude this paper by looking at the ring constructed in [3]. Given $m$, a nonzero monomial of $F$, define

$$
\begin{array}{r}
e^{\prime}(m)=\left(\max \left\{j_{k}\right\}\right) \text { (the number of times } Y_{\max j_{k}} \text { appears), } \\
\text { if } m \text { has a } Y \text { term, }
\end{array}
$$

$=0$, otherwise, 
and define

$$
e^{\prime}(f)=\max \left\{e^{\prime}\left(m_{i}\right)\right\},
$$

as in the definition of $e(f)$. Let $I^{\prime}$ be the ideal of $F$ generated by monomials $m$ such that $c(m)>\boldsymbol{\varepsilon}^{\prime}(m) \geq 1$, and let $R^{\prime}=F / I^{\prime}$. This is Osofsky's ring.

Remark. Let $S$ be the subring of $R^{\prime}$ consisting of those elements with zero constant term. Here we waive the condition that a ring must have unity. If $M$ is a faithful irreducible right $S$-module, then $M$ is a faithful irreducible right $R^{\prime}$-module, under the obvious action. Hence, if $S$ is primitive, then $R^{\prime}$ is primitive.

Theorem 4 (Osofsky [3]). $R^{\prime}$ is a prime semiprimitive ring and $Z\left(R^{\prime}\right)=(X)$.

Theorem 5. $R^{\prime}$ is not primitive.

Proof. Proof by contradiction. Suppose $R^{\prime}$ is primitive. Let $M$ be a proper right ideal of $R^{\prime}$, comaximal with every nonzero two-sided ideal. Then there exists $a \in(X)$ such that $a-1 \in M$. Choose $b$ large enough so that if $Y_{i}$ occurs in $a$, then $b>i$. There exists $b \in\left(Y_{b}\right)$ such that $b-1 \in M$. Let $n$ be a positive integer and consider $b a^{n}$. Let $m$ be any monomial in $b a^{n}$. Then $c(m) \geq n$ and $e^{\prime}(m) \leq e^{\prime}(b)$, by our choice of $h$. Thus, for sufficiently large $n, b a^{n}=0$, hence

$$
-1=(b-1) a^{n}+(a-1)\left[\sum_{i=0}^{n-1} a^{i}\right] \in M,
$$

a contradiction, since $M$ was assumed to be proper.

\section{REFERENCES}

1. C. Faith, Lectures in injective modules and quotient rings, Lecture Notes in Math., no. 49, Springer-Verlag, Berlin and New York, 1967. MR 37 \#2791.

2. E. Formanek, Group rings of free products are primitive, J. Algebra 26 (1973), 508-511.

3. B. Osofsky, A non-trivial ring with non-rational injective hull, Canad. Math. Bull. 10 (1967), 275-282. MR $35 \# 2930$.

DEPARTMENT OF MATHEMATICS, MCGILL UNIVERSITY, MONTREAL, QUEBEC, CANADA

Current address: Department of Mathematics, Carleton University, Ottawa, Ontario, Canada 